\title{
Directional Solidification of Ti6AI4V Ingots with an Electromagnetic Cold Crucible by Adjusting the Meniscus
}

\author{
Ruirun CHEN, ${ }^{*}$ Hongsheng DING, Jieren YANG, Jingjie GUO, Xinzhong LI, Yanqing SU and Hengzhi FU \\ School of Materials Science and Engineering, Harbin Institute of Technology, Harbin, Hei Longjiang, 150001 P.R. China. E-mail: \\ ruirunchen@hit.edu.cn, dinghsh@hit.edu.cn, doudoubest@126.com, guojj@hit.edu.cn, hitlxz@163.com, suyq@hit.edu.cn, \\ jiashan2000@163.com
}

(Received on Octover 21, 2011; accepted on February 16, 2012)

\begin{abstract}
Two different Ti6AI4V ingots were directionally solidified with an electromagnetic cold crucible using same parameters except the meniscus. Macrostructures of the two ingots were similar at the beginning of crystal growth, but became opposite at the end of crystal growth - the solid/liquid $(\mathrm{S} / \mathrm{L})$ interface is convex for one and concave for the other. Analysis of heat transfer during this cold crucible directional solidification showed that the meniscus influenced the macrostructure by changing the type of lateral heat transfer and the distance of heat conduction in axial direction. By increasing the mass of the meniscus, the lateral heat transfer changes from radiation to the combined action of radiation and heat conduction, and finally to heat conduction only. The distance of heat conduction increases at the same time. In order to achieve directional solidification, the meniscus must be kept at an optimal position in the electromagnetic field and must have appropriate size.
\end{abstract}

KEY WORDS: A. non-ferrous metals and alloys; C. casting; F. microstructure.

\section{Introduction}

Cold crucible technology has derived since the 1950's. Now it is becoming a new technique again because of the change of its configurations and the development of machining technology. It has been applied in many fields. ${ }^{1-3)}$ Cold crucible can be used not only for melting and shaping, but also for controlling the structure. The advantages of cold crucible are as follows: (1) Refractory metals can be melted. (2) Alloys of uniform composition can be created due to the strong stirring force of the electromagnetic field, (3) Metals can be melted free from interstitial contaminations. Directional solidification is a good method to obtain excellent mechanical performance in one direction and to expand fundamental studies on solidification theory.

Recently, directional solidification experiments were performed in optical floating zone furnaces or in resistance furnaces using ceramic crucibles. ${ }^{4-6)}$ Successful application of directional solidification is essential to produce complex shaped TiAl-based castings. Titanium is known as an element that can react with almost all ceramic mould materials. Long-term interactions between the melt and the ceramic mould due to low applied grain growth rate in directional solidification can result in contamination of the melt by ceramic inclusions, which will affect the microstructure and decrease mechanical properties of DS ingots.

Cold crucible directional solidification is an innovative process to combine the advantages of cold crucible, continuous casting and directional solidification. With this technique, directional solidification can be achieved without or with lower level of contamination, and can work with reac- tive materials, such as Ti-alloys, TiAl intermetallics, silicon and so on. However, there are many problems need to be solved in developing this technique. It is difficult to control the heat flow in cold crucible directional solidification because of the existence of water-cooling crucible wall. In order to achieve columnar grains of Titanium alloys, it is necessary to investigate this process from experimental and theoretical aspects. ${ }^{7,8)}$ Because of the electromagnetic force acting on the liquid metal, a meniscus (defined as the profile of the liquid metal that repelled from the crucible inner wall, as shown in Fig. 1) will be formed in cold crucible directional solidification. Therefore the position and the shape of the meniscus are the reflection of electromagnetic force, which will influence the induction heating and heat transfer process. Study of the meniscus is a keynote to understand the process of cold crucible directional solidification.

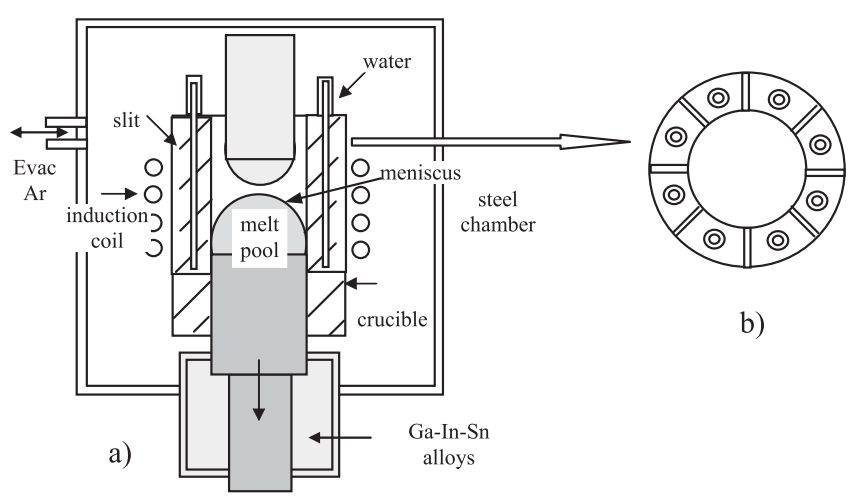

Fig. 1. Scheme of cold crucible diretional solidification equipment a) the whole apparatus b)cross section of the cold crucible. 


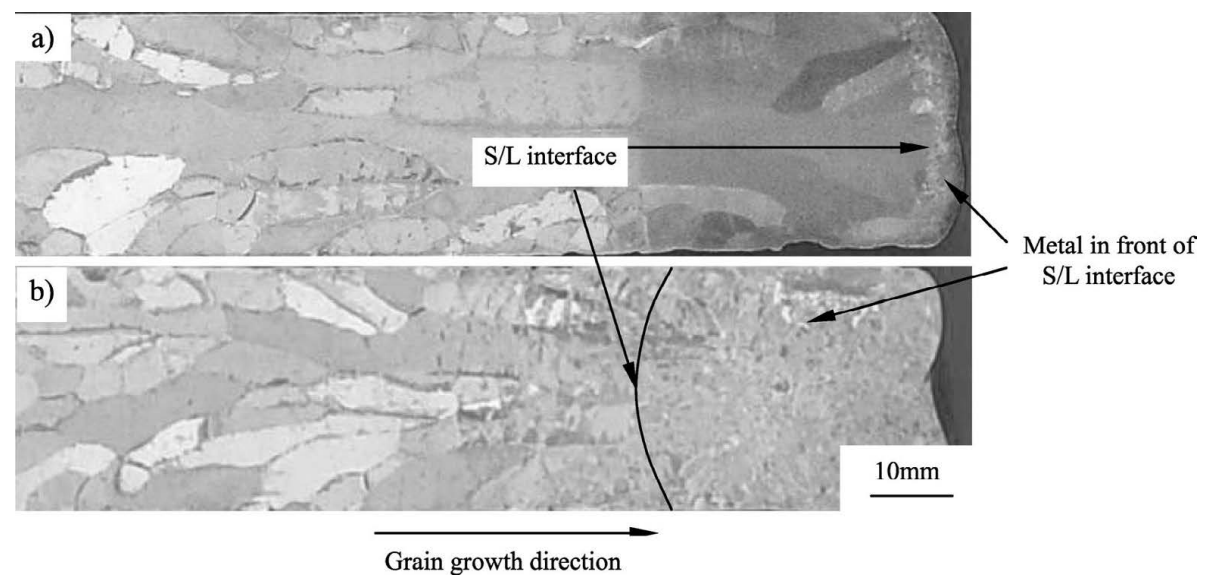

Fig. 2. Macrostructure of the ingots cast at $3 \mathrm{~mm} / \mathrm{min}$ velocity a) the ingot with a lower initial position and a small size meniscus $b$ ) the ingot with a higher initial position and a bigger size meniscus.

Available papers about the meniscus only present the interaction of the meniscus and the electromagnetic field. ${ }^{9-11)}$ The effect of the meniscus on macrostructures of the solidified ingots is rarely reported. In this paper, the mechanism of the meniscus influencing the macrostructure in cold crucible directional solidification is studied and revealed; some rules for obtaining the columnar grains of Ti6Al4V ingots are concluded.

\section{Experimental Methods}

The apparatus used in this work is a multi-functional electromagnetic cold crucible confinement and shaping equipment as shown in Fig. 1. This equipment consists of an inductive melting system, a controlling system, a cooling system and an air exhausting/argon filling system. The inner diameter of the cold crucible is $30 \mathrm{~mm}$. Ga-In-Sn liquid alloy was used for cooling the solidified titanium alloys ingots. Material used in the experiments was Ti6Al4V alloy. The charge in the crucible was induction heated when an AC current was supplied to the induction coil. After the charge was melted, some molten metal formed a meniscus when the pressure due to surface tension, hydrostatic pressure and electromagnetic pressure that acting on the molten metal were in mutual equilibrium. Movements of pulling solidified ingot and feeding raw materials rod started when the liquid metal has reached proper superheating temperature. A new ingot was then cast.

Results from previous work showed that the pulling velocity was the most important factor affecting the macrostructure in the cold crucible directional solidification. Other factors, such as power, coil turns and height of the primer have little effect on the macrostructure. Two different Ti6Al4V ingots were directionally solidified with pulling velocity of $3 \mathrm{~mm} / \mathrm{min}$. The different conditions for solidifying the two ingots were the initial position and the size of the meniscus, one has a lower position and a small size meniscus, the other has a higher position and a bigger size meniscus. The ingots were cut longitudinally, polished and etched.

\section{Results}

The macrostructure of the two ingots are shown in Fig. 2.
In Fig. 2(a) the grain growth direction in the central zone of the ingot parallels to the pulling direction, whereas the grain growth direction in the surface layer of the ingot departs from pulling direction with some angle. The grains grow towards the axis of the ingot and the $\mathrm{S} / \mathrm{L}$ interface is concave at the beginning of the solidification. In interim of the solidification, the grains grow parallel to the pulling direction and the $\mathrm{S} / \mathrm{L}$ interface is plane. The $\mathrm{S} / \mathrm{L}$ interface is convex at the end of solidification, and the volume of the liquid metal in front of the $\mathrm{S} / \mathrm{L}$ interface is very small. In Fig. 2(b), grain size is smaller in comparison with that in Fig. 2(a). All grains grow towards the axes of the ingot, and the volume of the liquid metal in front of the $\mathrm{S} / \mathrm{L}$ interface is large. Although the two ingots were directionally solidified at the same pulling velocity, they showed contrary grain growth and different volume of the liquid metal in front of the $\mathrm{S} / \mathrm{L}$ interface. The meniscus can be obtained by restoring the solidified part in front of the $\mathrm{S} / \mathrm{L}$ interface. There are some relationships between the meniscus and the $\mathrm{S} / \mathrm{L}$ interface, the meniscus has an important effect on the $\mathrm{S} / \mathrm{L}$ interface.

\section{Discussions}

Experimental results show that the meniscus has a very important effect on the macrostructure. Macrostructure tends to be directionally solidified when the volume of the meniscus is small. Macrostructure is decided by the $\mathrm{S} / \mathrm{L}$ interface and heat transfer, as it is discussed in the followings.

\subsection{Effect of the Meniscus on the $S / L$ Interface}

Effect of the meniscus on heat transfer can be sketched according to the process of cold crucible directional solidification, as shown in Fig. 3. In Fig. 3(a) image, the volume of meniscus is smaller and the three-phase line (where the line of the solid, the liquid and the gas meet each other) is at low position. Whereas in Fig. 3(b) image, the volume of meniscus is big and the three-phase line is at high position. A meniscus will be formed by a force balance between electromagnetic pressure, hydrostatic pressure and pressure due to surface tension.

Physical model of the $\mathrm{S} / \mathrm{L}$ interface shape is established based on the heat transfer in cold crucible directional solid- 


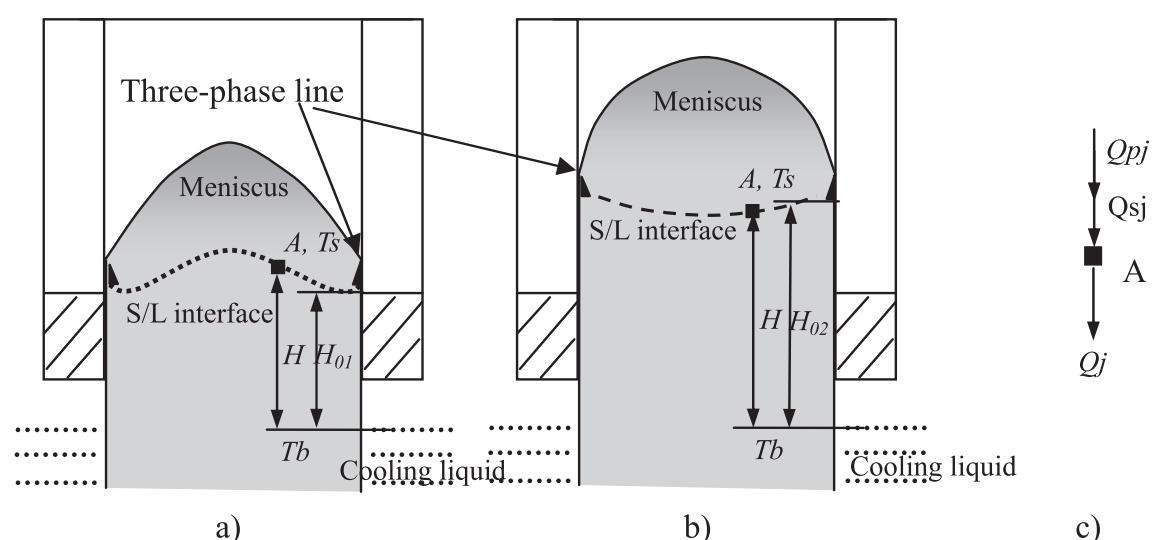

Fig. 3. Heat transfer when the meniscus at different position a) low position b) high position c) heat transfer in perpendicular direction of unit $\mathrm{A}$.

ification. In this model, the heat transfer is analyzed in perpendicular and horizontal directions, unit $\mathrm{A}$ is an arbitrary point of S/L interface. Because the crucible is round and axial symmetry, heat transfer of any unit of the $\mathrm{S} / \mathrm{L}$ interface is analyzed in perpendicular direction, as shown in Fig. 3. The heat transfer equation of unit $\mathrm{A}$ is Eq. (1), as shown below.

$$
Q_{p j}+Q_{s j}=Q_{j}
$$

$Q_{p j}$ is perpendicular part of induction heat of unit A,

$$
Q_{p j}=\Delta P_{j} \cdot \Delta t
$$

$Q_{s j}$ is perpendicular part of latent heat of unit A,

$$
Q_{s j}=L \cdot \rho \cdot d S \cdot V \cdot \Delta t \cdot \Delta j
$$

$Q j$ is perpendicular part of heat conduction of unit A,

$$
Q_{j}=\lambda \cdot d S \cdot \Delta t \cdot\left(T_{s}-T_{b}\right) / H
$$

where, $\Delta P_{j}$ - perpendicular part of effective power of unit A, W

$L —$ latent heat, $\mathrm{J} / \mathrm{kg}$

$V$ _ pulling velocity, $\mathrm{m} / \mathrm{s}$

$\rho$ density, $\mathrm{kg} / \mathrm{m}^{3}$

$d S-$ unit area of unit $\mathrm{A}, \mathrm{m}^{2}$

$\Delta j-$ perpendicular part coefficient of latent heat of unit A

$\lambda$ - thermal conductivity, $\mathrm{W} /(\mathrm{m} \cdot \mathrm{K})$

$\Delta t$ - unit time, $\mathrm{s}$

$T s$ s solidification temperature, $\mathrm{K}$

$\mathrm{Tb}$ - cooling liquid temperature, $\mathrm{K}$

$H$ is the length between unit $\mathrm{A}$ and cooling liquid level, $\mathrm{m}$

$H_{0}$ is the length of the skull bottom and cooling liquid level, $\mathrm{m}$

$$
\Delta P_{j} \cdot \Delta t+L \cdot \rho \cdot d S \cdot V \cdot \Delta t \cdot \Delta j=\lambda \cdot d S \cdot \Delta t \cdot\left(T_{s}-T_{b}\right) / H
$$

From Eq. (5), $H$ can be deduced as below,

$$
H=\frac{\lambda \cdot d S \cdot\left(T_{s}-T_{b}\right)}{\Delta P_{j}+L \cdot \rho \cdot d S \cdot V \cdot \Delta j}
$$

From Eq. (6), $H$ is decided not only by the pulling velocity, but also by the power and the temperature of the metal. In Fig. 3(a), when the meniscus volume is small, three-phase line keeps at a lower position, which means the position of meniscus is lower and $H_{01}\left(H_{01}\right.$ and $H_{2}$ are the height of the $\mathrm{S} / \mathrm{L}$ interface near the skull) is less, in this condition, $H>H_{01}$. Therefore, the $\mathrm{S} / \mathrm{L}$ interface is convex and grain size is larger and the macrostructure will be directionally solidified. However, in Fig. 3(b), when the meniscus volume is larger and three-phase line keeps at a higher position. $H_{0}$ is bigger so that $H<H_{02}$. Therefore the $\mathrm{S} / \mathrm{L}$ interface is concave (which results in that the macrostructure is not directionally solidified) and grain number becomes larger. Thus it can be concluded that the $\mathrm{S} / \mathrm{L}$ interface evolves from convex to plane, and then to concave, when $H_{0}$ increases with the increasing of meniscus volume.

Except pulling velocity, temperature gradient is another important factor in directional solidification. Induction heating is the heating source in cold crucible directional solidification, and the induction heating efficiency is decided by the position of the meniscus in the electromagnetic field. According to electromagnetic field distribution inside cold crucible, the induction heating efficiency is higher when the meniscus is located at the middle of the induction coil in axial direction. From above analysis, the temperature gradient will be increased when the meniscus is at appropriate position.

\subsection{Effect of the Meniscus on the Type of Lateral Heat Transfer}

Because of the lateral heat transfer, the grain growth direction will deviate from pulling direction. According to the theory of heat transfer, the quantity of lateral heat transfer is decided by the heat transfer coefficient and heat transfer area. When the meniscus is smaller, the liquid metal does not contact the crucible inner wall because the electromagnetic pressure is larger than the hydrostatic pressure, thus the type of heat transfer is heat radiation. When the meniscus increases, the hydrostatic pressure acting on the inner wall decreases because the electromagnetic pressure can counteract some of the hydrostatic pressure and the degree of the contact between the melt and the crucible inner wall becomes less. In that situation the type of heat transfer is a combination of heat conduction and heat radiation. The heat conduction coefficient decreases very much because of the electromagnetic pressure. Heat radiation is very low because of the induction heating induced from the electromagnetic field. So the heat transfer coefficient decreases to a low lev- 
el. In directional solidification, the lateral heat transfer of the zone near the $\mathrm{S} / \mathrm{L}$ interface is very important for obtaining a plane $\mathrm{S} / \mathrm{L}$ interface. The heat transfer area is small because the electromagnetic pressure can repel more liquid metal from the crucible inner wall when the meniscus is small. From above analysis, the lateral heat transfer is almost controlled or compensated when the meniscus is small. With increasing volume of the meniscus, the degree of the contact between the melt and the crucible inner wall becomes more. The type of heat transfer changes from the combination of heat conduction and heat radiation to heat conduction only and the heat transfer coefficient increases very much. The heat transfer area increases because the hydrostatic pressure is increased to more than the electromagnetic pressure. So it is difficult to get directional solidification with increasing of the meniscus. Analysis of heat transfer shows that the meniscus influences the macrostructure by changing the type of lateral heat transfer and the distance of heat conduction in axial direction. With the increase of the mass of meniscus, the type of lateral heat transfer changes from the radiation to combined action of radiation and heat conduction, to heat conduction only at last. The distance of heat conduction increases at the same time.

\subsection{Effect of the Electromagnetic Field on the Meniscus}

In Eq. (7), the meniscus is formed by a pressure balance from the electromagnetic force, hydrostatic pressure and the pressure due to surface tension.

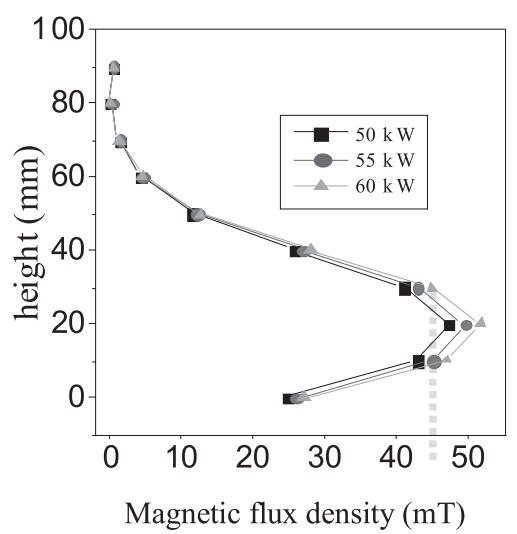

Fig. 4. Distribution of axial magnetic flux density in cold crucible under different power.

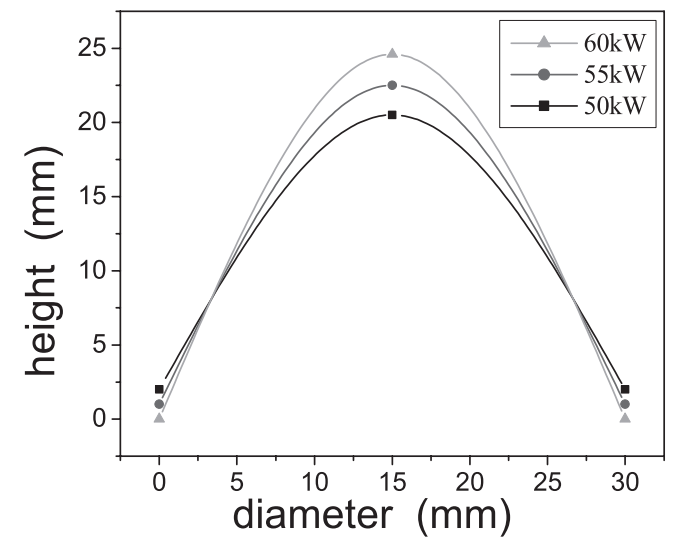

Fig. 5. Effects of the power on the position and the shape of the meniscus.

$$
\rho g h=P_{e}+P_{s}
$$

Where $\rho$ is the density of Ti6Al4V, $g$ is the gravity, $h$ is the height of the meniscus, $P_{e}$ is the magnetic pressure, $P_{s}$ is the pressure due to surface tension

From electromagnetism theory:

$$
P_{e}=B^{2} /(2 \mu)
$$

Where, $B$ is the magnetic flux density, $\mu$ is the magnetic permeability of Ti6Al4V.

Because the ingot is large enough, the pressure due to surface tension can be neglected. The relation of the height of the meniscus and the magnetic induction is

$$
h_{x}=B^{2} /(2 \mu \rho g)=\mu H^{2} /(2 \rho g)
$$

Where, $H$ is the magnetic field intensity, Eq. (9) shows that the height of the meniscus is proportion to the square of the magnetic field intensity. Magnetic fields in the crucible under different power are calculated by mutual inductance coupling model, and the calculation results are shown in Fig. 4. Magnetic field intensity increases with the increasing of the power.

Because the existence of the slits in the cold crucible, the electromagnetic field is not even at the same height, this will lead to the uneven of the three-phase line. In order to simplify the process of analyzing, the calculation conditions are supposed ideal. Suppose the height of the meniscus is even and the magnetic flux density that forms this meniscus is $45 \mathrm{mT}$. According to Eq. (9), the heights of three-phase line are 15.4, 10.5, $8.9 \mathrm{~mm}$ respectively, as shown in Fig. 4 by the dashed line. This means that the position of three-phase line decreases with the increasing of the power.

Suppose the three-phase line is at the position of the maximum of the magnetic flux density, the height of the meniscus can be calculated according to Eq. (9), and a schematic of meniscus under different power is achieved, as shown in Fig. 5. Increasing the power, the meniscus height increases

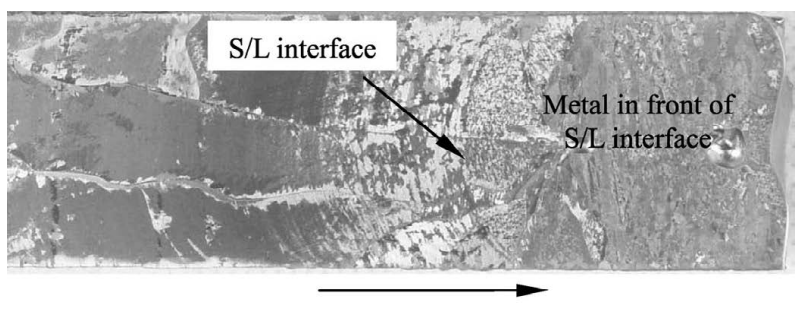

Grain growth direction

Fig. 6. The change of macrostructure with the increasing of the volume of the meniscus.

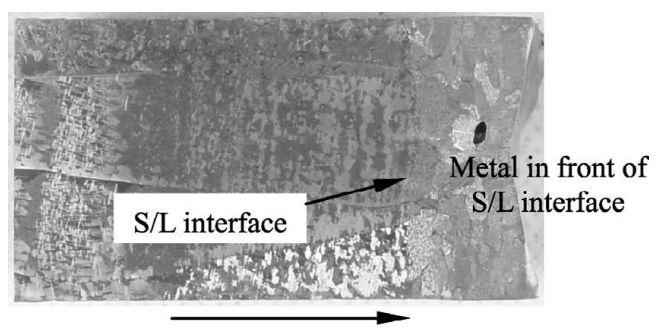

Grain growth direction

Fig. 7. The ingot with columnar grains and plane $S / L$ interface. 
and the three-phase line descends, which means $H_{0}$ is reduced, and the ingot tends to be solidified directionally.

From the above analysis, it can be concluded that in order to directionally solidify the Ti6Al4V ingot, the meniscus must have an appropriate volume and the three-phase line should be kept at an appropriate position, which requires optimized technical parameters.

In order to verify this conclusion, an experiment of increasing the volume of meniscus by continuous increasing feeding materials was carried out, and the result is shown in Fig. 6. It can be seen that the $\mathrm{S} / \mathrm{L}$ interface evolves from plane to concave, which is in well agreement with the above analysis. Another experiment was done with an appropriate meniscus volume, the $\mathrm{S} / \mathrm{L}$ interface is plane and the grains are columnar, as shown in Fig. 7, which is a good example of cold crucible directionally solidifying of Ti6Al4V alloys.

\section{Conclusions}

Grain size increases and the grain growth direction tends to be parallel to the pulling direction with the decrease of the volume of the meniscus. The macrostructure will be directionally solidified when the meniscus is kept an appropriate position and volume. The position of meniscus is decided by the volume of the liquid dome when other parameters are not changed. The position ascends with the increase of the volume of liquid dome. The meniscus height is proportion to the square of the magnetic flux density. The meniscus height increases and the height of the three-phase line decreases with the increase of the power. With increasing of the mass of meniscus, the type of lateral heat transfer changes from the radiation to combined action of radiation and heat conduction, and to heat conduction only finally. The distance of heat conduction increases at the same time.

\section{Acknowledgements}

This research was funded by National Basic Research Program of China (No. 2011CB605504), State Key Laboratory of Materials Processing and Die \& Mould Technology, Huazhong University of Science and Technology (2010-P10).

\section{REFERENCES}

1) B. G. JIin, Q. Wang, A. Gao, Y. Q. Yu and J. C. He: ISIJ Int., 49 (2009), 44.

2) H. S. Ding, R. R. Chen, J. J. Guo, W. S. Bi, H. Z. Fu and C. J. Zhang: China Foundry, 1 (2004), 28.

3) A. Morita, H. Fukui, H. Tadano, S. Hayashi, J. Hasegawa and M. Niinomi: Mater. Sci. Eng. A, 280 (2000), 208.

4) A. P. Silva, J. E. Spinelli, N. Mangelinck-Noël and A. Garcia: Mater. Design, 31 (2010), 4584.

5) P. R. Cha, Y. S. Hwang, Y. J. Oh, S. H. Chung and J. K. Yoon: ISIJ Int., 36 (1996), 1157.

6) P. R. Cha, Y. S. Hwang, H. S. Nam, S. H. Chung and J. K. Yoon: ISIJ Int., 38 (1998), 403.

7) R. R. Chen, H. S. Ding, J. J. Guo, F. Z. Liang, Z. Q. Zhang, W. S. Bi, L. Liu and H. Z. Fu: J. Mater. Sci. Technol., 17 (2009), 482.

8) R. R. Chen, H. S. Ding, J. J. Guo, W. S. Bi and H. Z. Fu: China Foundry, 8 (2007), 190.

9) F. Negrini, M. Fabbri, M. Zuccarini, E. Takeuchi and M. Tani: Energ. Convers. Manage., 41 (2000), 687.

10) T. Tanaka, K. Kurita and A. Kuroda: ISIJ Int., 31 (1991), 350.

11) Y. Kawase and T. Yoshida: IEEE Trans. Magn., 35 (1999), 1889.

12) M. Hanao, M. Kawamoto and A. Yamanaka: ISIJ Int., 49 (2009), 365 